\title{
Laboratory Astrophysics with COSmIC: Interstellar and Planetary Applications
}

\author{
Farid Salama $^{1}$, Cesar S. Contreras ${ }^{1,2}$, \\ Ella Sciamma-O'Brien ${ }^{1,2}$ and Salma Bejaoui ${ }^{1,3}$ \\ ${ }^{1}$ NASA Ames Research Center, Moffett Field, CA \\ ${ }^{2}$ Bay Area Environmental Research Institute, Petaluma, CA \\ ${ }^{3} \mathrm{NPP}$, Oak Ridge Associated Universities, TN \\ email: farid.salama@nasa.gov
}

\begin{abstract}
We discuss the capabilities of the laboratory facility, COSmIC, that was developed to generate, process and analyze interstellar, circumstellar and planetary analogs in the laboratory
\end{abstract}

Keywords. COSmIC, Interstellar, Circumstellar, Planetary, Laboratory, Analogs

COSmIC is a simulation chamber for the study of molecules, ions and grains under the low temperature and high vacuum conditions that are required to simulate space environments (Salama (2008)). COSmIC is equipped with a Pulsed Discharge Nozzle (PDN) expansion that generates a plasma in a supersonic jet expansion coupled to highsensitivity in situ diagnostics: cavity ring down spectroscopy (CRDS), laser induced fluorescence (LIF) and Reflectron time-of-flight mass spectrometry (ReTOF-MS; Ricketts et al. (2011)). Typical applications of COSmiC are illustrated below:

Interstellar applications: CRDS spectra of PAH molecules prepared in a cold jet expansion in COSmIC were used in astronomical surveys of PAHs in translucent clouds, leading to column densities estimates (Salama et al. (2011)). Laser-induced fluorescence (LIF) studies have been initiated to study PAH molecules emission for comparison with the extended red emission (ERE) and cometary spectra ((Bejaoui et al. 2015).

Circumstellar applications: Experiments with COSmIC use the cold plasma sources to explore the formation processes of carbon molecules and nano-sized dust grains in the circumstellar outflows of late carbon stars (Contreras \& Salama (2013)).

Planetary applications: The Titan Haze Simulation (THS) experiment on COSmIC is used to study aerosol formation in Titan's hazy atmosphere. $N_{2}$-hydrocarbon gas mixtures are injected in the plasma in order to monitor the evolution of the chemical growth and probe the early stages of aerosol formation at Titan-like temperature $(150 \mathrm{~K}$; Sciamma-O'Brien et al. (2014)). Grains are also produced that can be jet deposited onto various substrates providing a good simulation of probe descent into Titan's atmosphere.

\section{References}

Bejaoui, S., Salama, F., \& Sciamma-O'Brien, E. 2015, this volume

Contreras, S. C. \& Salama, F. 2013, The Astrophys. J. Suppl. Ser., 208, 6

Ricketts, C. L., Contreras, S. C., Walker, R., \& Salama, F. 2011, Int. J. Mass Spec., 300, 26

Salama, F. 2008, in: S. Kwok \& S. Sandford (eds.), Organic Matter in Space, IAU Symposium 251 (Cambridge University Press), Vol. 4, p. 357

Salama, F., Galazutdinov, G. A., Krelowski, J., Biennier, L., Beletsky, Y., and In-Ok Song 2011 The Astrophysical Journal, 728, 154

Sciamma-O'Brien, E., Ricketts, C. L., \& Salama, F. 2014, Icarus, 243, 325 